\title{
Oscillation for higher order differential equations with a middle term
}

\author{
Miroslav Bartušek', Zuzana Došlá ${ }^{*}$ and Mauro Marini² \\ Dedicated to Professor I Kiguradze.
}

"Correspondence:

dosla@math.muni.cz

${ }^{1}$ Department of Mathematics and

Statistics, Faculty of Science,

Masaryk University, Kotlářská 2,

Brno, 611 37, Czech Republic

Full list of author information is

available at the end of the article

\begin{abstract}
We study the existence of bounded oscillatory solutions for a higher order differential equation, considered as a perturbation of an associated linear equation. Jointly with this, we study the nonexistence of solutions vanishing at infinity and, as an application, we obtain in the linear case an asymptotic equivalency criterion.
\end{abstract}

\section{Introduction}

Consider the higher order nonlinear differential equation with a middle term

$$
x^{(n)}(t)+q(t) x^{(n-2)}(t)+r(t) f(x(t))=0,
$$

where $n \geq 3$ and $t \geq 0$. Throughout the paper, we assume that $f \in C(\mathbb{R})$ such that $f(u) u>0$ for $u \neq 0, q \in C^{1}[0, \infty), r \in C[0, \infty)$, and

$$
q(t) \geq q_{0}>0, \quad \int_{0}^{\infty}\left|q^{\prime}(t)\right| d t<\infty .
$$

Hence, $q$ is bounded from above and $\lim _{t \rightarrow \infty} q(t)=q_{\infty}<\infty$. Note that the function $r$ may change sign.

By a solution of (1) we mean a function $x$ differentiable up to order $n$ which satisfies (1) on $\left[T_{x}, \infty\right), T_{x} \geq 0$. A solution of (1) is said to be proper if $\sup \{|x(t)|: t \geq T\}>0$ for any $T \geq T_{x}$. As usual, a solution $x$ of (1) is said to be oscillatory if $x$ changes sign for large $t$.

The aim of this paper is to study (1) as a perturbation of the linear differential equation

$$
y^{(n)}(t)+q(t) y^{(n-2)}(t)=0
$$

An important role in our approach is played by the relationship between solutions of (2) and those of the second order linear equation

$$
h^{\prime \prime}(t)+q(t) h(t)=0 .
$$

This approach is mainly motivated by previous results, obtained by Kiguradze in [1] for the special case $q(t) \equiv 1$, i.e., for the equation

$$
x^{(n)}(t)+x^{(n-2)}(t)+r(t) f(x(t))=0 .
$$

@2014 Bartušek et al.; licensee Springer. This is an Open Access article distributed under the terms of the Creative Commons Attribution License (http://creativecommons.org/licenses/by/2.0), which permits unrestricted use, distribution, and reproduction in any medium, provided the original work is properly cited. 
It was shown in [1] that, if $r$ is positive and sufficient large in some sense, then for $n$ even every proper solution of (4) is oscillatory. Moreover, for $n$ odd every proper solution of (4) is oscillatory, or is vanishing at infinity together with its derivatives, or admits the asymptotic representation

$$
x(t)=c(1+\sin (t-\varphi))+\varepsilon(t)
$$

where $c, \varphi$ are suitable constants and $\varepsilon$ is a continuous function for $t \geq 0$ which vanishes at infinity. According to [1], in this case (4) is said to have property $A^{\prime}$.

The existence of solutions vanishing at infinity, and the closely related problem on existence of bounded oscillatory solutions, has attracted the attention in many papers, see, e.g. the monograph [2] and references therein. In particular, nonlinear equations with middle term have been investigated in many directions, as a perturbation of an associated linear equation. For third and fourth order equations, we refer especially to [3-5], in which the property A, or its generalizations, has been studied and to [6-10] for oscillation problems. In particular, in [5] a good and detailed discussion of known oscillation criteria is given as well.

The higher order equations of type (1) have been studied in [11-13]. More precisely, in $[11,12]$ the general equation

$$
x^{(n)}(t)+\sum_{j=0}^{n-1} q_{j}(t) x^{(j)}(t)+r(t)|x(t)|^{\lambda} \operatorname{sgn} x(t)=0, \quad \lambda>1,
$$

has been considered. In [11] sufficient conditions are obtained for the existence of solutions, which are equivalent to a polynomial. In [12] a criterion is given for existence of nonoscillatory solutions with non-zero limit at infinity. Moreover, for $n$ even, an oscillation result is obtained too. In [13], (1) is studied as a perturbation of (2), under the assumption $(\mathrm{H})$. Finally, the case of higher order equations with forcing term has been considered in the recent papers [14-16], see also the last section.

Observe that if $q$ is a positive constant, then (2) has oscillatory and bounded solutions not vanishing at infinity. If $q$ is not constant and $(\mathrm{H})$ is satisfied, then these properties remain to hold for the second order equation (3), see, e.g. [17, Chapter 6], or [18, Theorem 2]. Thus, it is natural to ask under which assumptions these properties are valid also for (2) (when $q$ is not constant) and for the more general case (1).

In this paper, we answer both these questions. In particular, our main result yields the existence of oscillatory solutions of (1), which are bounded and not vanishing at infinity. Our results complete recent ones in [13, Corollary 1, Corollary 3], extend similar ones in [1, Theorem 1.3, Theorem 1.4], which are proved for (4), and generalize ones in [3, 10], which are stated for the particular cases $n=3,4$.

In Section 3 we study the problem of the nonexistence of solutions vanishing at infinity of (1). This result will be employed in Section 4 and Section 5, namely to prove the existence of oscillatory solutions of (2) (Section 4) and the uniqueness of solutions of (1), which have the same asymptotics as solutions of (2) (Section 5).

We close the paper with an application that concerns the influence of the perturbing term $r$ on the change of the oscillatory character, passing from (2) to the linear equation

$$
x^{(n)}(t)+q(t) x^{(n-2)}(t)+r(t) x(t)=0, \quad n \geq 3 .
$$


More precisely, we will give conditions under which (2) and (5) are asymptotically equivalent. Some suggestions for further researches complete the paper.

\section{Preliminaries}

We start with some basic properties of solutions of (3), which will be useful in the sequel. Obviously, assumption $q(t) \geq q_{0}>0$ implies that (3) is oscillatory. Moreover, the following holds.

Lemma 1 Let $(\mathrm{H})$ hold and let $u$ be a nontrivial solution of (3). Then $u$ is oscillatory and

$$
0<\limsup _{t \rightarrow \infty}|u(t)|<\infty, \quad 0<\limsup _{t \rightarrow \infty}\left|u^{\prime}(t)\right|<\infty .
$$

Proof Since $q$ is of bounded variation for $t \geq 0$, all solutions of (3) are bounded together with their derivatives, see e.g. [18, Theorem 2]. Moreover, the function $q$ can be represented as $q(t)=a(t) / b(t)$, where $a, b$ are positive nonincreasing and differentiable functions such that $\lim _{t \rightarrow \infty} a(t)>0$ and $\lim _{t \rightarrow \infty} b(t)>0$, for details see [19, Lemma 5.4.1]. Hence, taking $t_{0}$ such that $u^{\prime}\left(t_{0}\right)=0$ and applying [13, Lemma 2], we get the assertion.

Let $u, v$ be two linearly independent solutions of (3) with Wronskian equal to 1 . Set

$$
w(s, t)=u(s) v(t)-u(t) v(s), \quad \zeta(s, t)=\frac{\partial}{\partial t} w(s, t) .
$$

Then $w$ and $\zeta$ are bounded in $[0, \infty) \times[0, \infty)$.

Equations (2) and (3) are strictly related. When $q(t) \equiv 1$, a basis of the space of solutions of (2) is given by

$$
t^{j}, \quad j=0,1, \ldots, n-3, \quad \sin t, \quad \cos t .
$$

In the general case, that is, when $q$ is not constant, it is easy to see that a basis of the space of solutions of (2) is given by

$$
t^{j}, \quad j=0,1, \ldots, n-3, \quad \Gamma_{u}, \quad \Gamma_{v},
$$

where

$$
\Gamma_{u}=\int_{0}^{t}(t-s)^{n-3} u(s) d s, \quad \Gamma_{v}=\int_{0}^{t}(t-s)^{n-3} v(s) d s
$$

and $u, v$ are two independent solutions of (3). The question whenever the sets (7) and (8) are, roughly speaking, close as $t \rightarrow \infty$ is considered in Section 5 .

We close this section by recalling the main result from [13], which plays a crucial role in our further consideration. Let

$$
\bar{f}(u)=\max \{|f(v)|:-u \leq v \leq u\} .
$$

The symbol $g_{1}=O\left(g_{2}\right)$ as $t \rightarrow \infty$ means, as usual, that there exists a constant $M$ such that in a neighborhood of infinity

$$
\left|g_{1}(t)\right| \leq M\left|g_{2}(t)\right|
$$

The following holds. 
Theorem A ([13, Theorem 1]) Assume $n \geq 3$. Let for any positive constant $\mu$ and for some $j \in\{0, \ldots, n-3\}$

$$
\int_{0}^{\infty} t^{n-3} \bar{f}\left(\mu t^{j}\right)|r(t)| d t<\infty .
$$

Then, for any solution $y$ of (2) such that $y(t)=O\left(t^{j}\right)$ as $t \rightarrow \infty$, there exists a solution $x$ of (1) such that for large $t$

$$
x^{(i)}(t)=y^{(i)}(t)+\varepsilon_{i}(t), \quad i=0, \ldots, n-1,
$$

where $\varepsilon_{i}$ are functions of bounded variation for large $t$ and $\lim _{t \rightarrow \infty} \varepsilon_{i}(t)=0, i=0, \ldots, n-1$.

Remark 1 If $f$ satisfies in a neighborhood $I$ of zero

$$
|f(u)| \leq K|u|
$$

for some $K>0$, then every nontrivial solution of (1) is proper. This follows from [1, Theorem 11.5] with $h(t)=q_{\max }+K|r(t)|$ and $\omega(u)=u$ for $u \in I$.

\section{Solutions vanishing at infinity}

Our main result in this section is concerned with the nonexistence of solutions vanishing at infinity.

Theorem 1 Let $n \geq 3, f \in C^{1}(\mathbb{R})$ and

$$
\int_{0}^{\infty} t^{n-3}|r(t)| d t<\infty
$$

Then (1) does not have nontrivial solutions $x$ (oscillatory or nonoscillatory) satisfying

$$
\lim _{t \rightarrow \infty} x(t)=0 .
$$

To prove Theorem 1, some lemmas will be needed. The first result concerns the linear nonhomogeneous equation

$$
z^{(n)}(t)+q(t) z^{(n-2)}(t)=F(t)
$$

where $F \in C[0, \infty)$.

Lemma 2 Assume $n \geq 3$. Let $F \in C[0, \infty)$ satisfy

$$
\int_{0}^{\infty} t^{n-3}|F(t)| d t<\infty
$$

Then

$$
z(t)=(-1)^{n} \int_{t}^{\infty} \int_{\tau_{n-2}}^{\infty} \cdots \int_{\tau_{1}}^{\infty} w\left(s, \tau_{1}\right) F(s) d s d \tau_{1} \cdots d \tau_{n-2},
$$


where $w$ is defined by (6), is a solution of (15). Moreover, there exists $M>0$ such that for $t \geq 0$

$$
|z(t)| \leq M \int_{t}^{\infty} s^{n-3}|F(s)| d s .
$$

Proof If $F(t) \equiv 0$ for large $t$, then the assertion holds. Thus, assume that $F \not 0$ for large $t$.

First, we prove that $z$ is well defined. Choose $\bar{t}$ large so that

$$
\frac{1}{q_{0}} \int_{\bar{t}}^{\infty}\left|q^{\prime}(s)\right| d s=\ell_{1}<1
$$

Let $m$ be a constant such that $|w(s, \tau)| \leq m,|\zeta(s, \tau)| \leq m$ for $s \geq \tau \geq \bar{t}$. From (16) we have

$$
\begin{aligned}
& \left|\int_{\bar{t}}^{\infty} \int_{\tau_{n-3}}^{\infty} \cdots \int_{\tau_{1}}^{\infty} w\left(s, \tau_{1}\right) F(s) d s d \tau_{1} \cdots d \tau_{n-3}\right| \\
& \quad \leq m \int_{\bar{t}}^{\infty} \cdots \int_{\tau_{1}}^{\infty}|F(s)| d s d \tau_{1} \cdots d \tau_{n-3} \leq m \int_{\bar{t}}^{\infty} s^{n-3}|F(s)| d s<\infty .
\end{aligned}
$$

Hence, for fixed $T>\bar{t}$ the function

$$
\alpha(t)=(-1)^{n} \int_{t}^{T} \int_{\tau_{n-2}}^{\infty} \cdots \int_{\tau_{1}}^{\infty} w\left(s, \tau_{1}\right) F(s) d s d \tau_{1} \cdots d \tau_{n-2}
$$

is well defined for $t \geq \bar{t}$. In particular, we have

$$
\alpha^{(n-3)}(t)= \begin{cases}-\int_{t}^{\infty} \int_{r}^{\infty} F(s) w(s, r) d s d r & \text { if } n>3 \\ -\int_{t}^{T} \int_{r}^{\infty} F(s) w(s, r) d s d r & \text { if } n=3\end{cases}
$$

and for $n \geq 3$

$$
\begin{aligned}
& \alpha^{(n-2)}(t)=\int_{t}^{\infty} F(s) w(s, t) d s, \\
& \alpha^{(n-1)}(t)=\int_{t}^{\infty} F(s) \zeta(s, t) d s, \\
& \alpha^{(n)}(t)=-F(t)-q(t) \alpha^{(n-2)}(t) .
\end{aligned}
$$

Consider the function

$$
\gamma(t)=\alpha^{(n-1)}(t)+q(t) \alpha^{(n-3)}(t), \quad \bar{t} \leq t .
$$

Then

$$
\gamma^{\prime}(t)=\alpha^{(n)}(t)+q(t) \alpha^{(n-2)}(t)+q^{\prime}(t) \alpha^{(n-3)}(t)=-F(t)+q^{\prime}(t) \alpha^{(n-3)}(t) .
$$

Integrating this equality on $(t, T)$, we get

$$
\gamma(t)=\int_{t}^{T} F(s) d s-\int_{t}^{T} q^{\prime}(s) \alpha^{(n-3)}(s) d s+\gamma(T) .
$$


From this, (20), and (21), we obtain

$$
q(t) \alpha^{(n-3)}(t)=\int_{t}^{T} F(s) d s-\int_{t}^{T} q^{\prime}(s) \alpha^{(n-3)}(s) d s-\int_{t}^{\infty} F(s) \zeta(s, t) d s+\gamma(T)
$$

or, from (18),

$$
\left|\alpha^{(n-3)}(t)\right| \leq \frac{1}{q_{0}}\left(\int_{t}^{T}|F(s)| d s+m \int_{t}^{\infty}|F(s)| d s+|\gamma(T)|\right)+\ell_{1} \max _{t \leq s \leq T}\left|\alpha^{(n-3)}(s)\right| .
$$

In view of (16), we have $\left|\alpha^{(n-3)}(T)\right|=0$ or $\left|\alpha^{(n-3)}(T)\right| \leq m \int_{T}^{\infty} s|F(s)| d s$ according to $n=3$ or $n>3$, respectively. Moreover,

$$
\left|\alpha^{(n-1)}(T)\right| \leq m \int_{T}^{\infty}|F(s)| d s \quad \text { for any } n \geq 3
$$

Hence, from (21) we obtain

$$
\lim _{T \rightarrow \infty}|\gamma(T)|=0, \quad n \geq 3
$$

For $n=3$ we have $|\alpha(T)|=0$ and

$$
|\gamma(T)|=\left|\int_{T}^{\infty} F(s) \zeta(s, t) d s\right| \leq m \int_{t}^{\infty}|F(s)| d s .
$$

From (22), in case $n>3$ using (23) and letting $T \rightarrow \infty$, and in case $n=3$ using (24) we get

$$
\left|\alpha^{(n-3)}(t)\right| \leq \ell_{2} \int_{t}^{\infty}|F(s)| d s+\ell_{1} \sup _{s \geq t}\left|\alpha^{(n-3)}(s)\right|, \quad n \geq 3,
$$

where $\ell_{2}=(1+2 m) / q_{0}$. Thus

$$
\begin{aligned}
\left|\alpha^{(n-3)}(t)\right| & \leq \sup _{\tau \geq t}\left|\alpha^{(n-3)}(\tau)\right| \\
& \leq \sup _{\tau \geq t}\left(\ell_{2} \int_{\tau}^{\infty}|F(s)| d s+\ell_{1} \sup _{s \geq \tau}\left|\alpha^{(n-3)}(s)\right|\right) \\
& \leq \ell_{2} \int_{t}^{\infty}|F(s)| d s+\ell_{1} \sup _{\tau \geq t}\left|\alpha^{(n-3)}(\tau)\right|
\end{aligned}
$$

or

$$
\left(1-\ell_{1}\right) \sup _{s \geq t}\left|\alpha^{(n-3)}(s)\right| \leq \ell_{2} \int_{t}^{\infty}|F(s)| d s,
$$

that is

$$
\left|\alpha^{(n-3)}(t)\right| \leq \frac{\ell_{2}}{1-\ell_{1}} \int_{t}^{\infty}|F(s)| d s .
$$

When $n>3$, since $\lim _{t \rightarrow \infty}\left|\alpha^{(i)}(t)\right|=0$ for $i=1, \ldots, n-3$, using (25) we get

$$
\left|\alpha^{(i)}(t)\right| \leq \int_{t}^{\infty}\left|\alpha^{(i+1)}(s)\right| d s \leq \frac{\ell_{2}}{1-\ell_{1}} \int_{t}^{\infty} \frac{(s-t)^{n-3-i}}{(n-3-i) !}|F(s)| d s
$$


From this, taking into account $\alpha(T)=0$, we obtain

$$
|\alpha(t)|=\left|\int_{t}^{T} \alpha^{\prime}(s) d s\right| \leq M \int_{t}^{\infty} s^{n-3}|F(s)| d s
$$

where $M=\ell_{2} /\left(1-\ell_{1}\right)(n-3)$ !. If $n=3$, then (26) holds by (25).

Now, for the sake of clarity, denote by $\alpha(t, T)$ the function $\alpha$ given in (19). Fix $\tilde{t} \geq \bar{t}$. In virtue of $\alpha(\tilde{t}, T)=\alpha\left(\tilde{t}, T_{0}\right)+\alpha\left(T_{0}, T\right)$ we have

$$
\left|\alpha\left(\tilde{t}, T_{1}\right)-\alpha\left(\tilde{t}, T_{2}\right)\right|=\left|\alpha\left(T_{0}, T_{1}\right)-\alpha\left(T_{0}, T_{2}\right)\right|
$$

where $T_{i} \geq \tilde{t}, i=0,1,2$. Thus for $T_{0}$ large so that

$$
M \int_{T_{0}}^{\infty} s^{n-3}|F(s)| d s<\frac{\varepsilon}{2}
$$

and from (26) we obtain

$$
\left|\alpha\left(T_{0}, T_{1}\right)-\alpha\left(T_{0}, T_{2}\right)\right| \leq\left|\alpha\left(T_{0}, T_{1}\right)\right|+\left|\alpha\left(T_{0}, T_{2}\right)\right|<\varepsilon
$$

for any $T_{1}>T_{0}, T_{2}>T_{0}$. Using the Cauchy criterion, in virtue of (26), there exists the finite limit

$$
\lim _{T \rightarrow \infty} \alpha(\tilde{t}, T)=\alpha_{\infty}, \quad\left|\alpha_{\infty}\right|<\infty
$$

Hence, $z$ is well defined. A direct computation shows that $z$ is a solution of (15).

It remains to prove that $z$ satisfies the estimation (17). Using (26) we have

$$
|z(t)|=\lim _{T \rightarrow \infty}|\alpha(t)| \leq M \int_{t}^{\infty} s^{n-3}|F(s)| d s
$$

which yields (17).

Remark 2 Under the stronger assumption $\int_{0}^{\infty} t^{n-2}|F(t)| d t<\infty$, Lemma 2 follows with a standard calculation.

Lemma 3 Let $n \geq 3$ and $y \in C^{(n-1)}[T, \infty), T \geq 0$, be such that

$$
\lim _{t \rightarrow \infty} y(t)=0 \quad \text { and } \quad y^{(n-1)} \quad \text { is bounded on }[T, \infty)
$$

Then

$$
\lim _{t \rightarrow \infty} y^{(i)}(t)=0 \quad \text { for } i=0,1, \ldots, n-2
$$

Proof Let $b \in(T, \infty)$. Since $y$ and $y^{(n-2)}$ are bounded, we can apply [2, Lemma 5.2] with $m=n-1$ and we obtain

$$
\max _{t \in[T, b]}\left|y^{(i)}(t)\right| \leq \frac{C_{1}}{(b-T)^{i}} \max _{t \in[T, b]}|y(t)|+C_{2}\left(\max _{t \in[T, b]}|y(t)|\right)^{\frac{i}{n-1}}\left(\max _{t \in[T, b]}\left|y^{(n-1)}(t)\right|\right)^{\frac{n-1-i}{n-1}}
$$


where $i=1,2, \ldots, n-2$ and $C_{1}, C_{2}$ are suitable positive constants. Passing $b$ to $\infty$, there exists a positive constant $C_{3}$ such that

$$
\sup _{t \in[T, \infty)}\left|y^{(i)}(t)\right| \leq C_{3}\left(\sup _{t \in[T, \infty)}|y(t)|\right)^{\frac{i}{n-1}}
$$

which gives the assertion, because $\lim _{t \rightarrow \infty} y(t)=0$.

The next auxiliary result is a Gronwall type lemma, which proof is elementary and so it is omitted.

Lemma 4 Let $g$ and $\Psi$ be nonnegative continuous functions for $t \geq t_{0} \geq 0$ such that $\Psi$ and $\Psi \mathrm{g}$ belong to $L^{1}\left[t_{0}, \infty\right)$. If

$$
g(t) \leq A+\int_{t}^{\infty} \Psi(s) g(s) d s \quad\left(t \geq t_{0}\right)
$$

for some nonnegative constant $A$, then

$$
g(t) \leq A \exp \left(\int_{t}^{\infty} \Psi(s) d s\right) \quad\left(t \geq t_{0}\right)
$$

In particular, if $A=0$, then $g(t)=0$ for each $t \in\left[t_{0}, \infty\right)$.

Proof of Theorem 1 Assume that there exists a nontrivial solution $x$ of (1) defined on $\left[T_{x}, \infty\right)$ and satisfying (14).

Let $u, v$ be linearly independent solutions of (3) with Wronskian 1 . By Lemma 1 ,

$$
u, \quad u^{\prime}, \quad v, \quad v^{\prime} \text { are bounded. }
$$

Hence, $w(s, t)$, defined by (6), is bounded. Put $h(t)=x^{(n-2)}(t)$. Then $h$ is a solution of the second order equation

$$
h^{\prime \prime}(t)+q(t) h(t)=F(t)
$$

where $F(t)=-r(t) f(x(t))$. Since $r \in L^{1}[0, \infty)$, from (14) and (28), we obtain

$$
\int_{T_{x}}^{\infty}|u(s) F(s)| d s<\infty, \quad \int_{T_{x}}^{\infty}|v(s) F(s)| d s<\infty .
$$

Taking into account (28), (29) and using the variation constant formula, we have

$$
h(t)=x^{(n-2)}(t)=C_{1} u(t)+C_{2} v(t)-\int_{t}^{\infty} w(s, t) F(s) d s,
$$

where $C_{1}, C_{2}$ are suitable constants.

Thus

$$
x^{(n-1)}(t)=C_{1} u^{\prime}(t)+C_{2} v^{\prime}(t)-\int_{t}^{\infty} \zeta(s, t) F(s) d s .
$$


From this and (28), we see that $x^{(n-1)}$ is bounded on $\left[T_{x}, \infty\right)$. By Lemma 3 we obtain

$$
\lim _{t \rightarrow \infty} x^{(i)}(t)=0, \quad i=0,1, \ldots, n-2
$$

Hence, from (30)

$$
\lim _{t \rightarrow \infty}\left[C_{1} u(t)+C_{2} v(t)\right]=0
$$

We claim that $C_{1}=C_{2}=0$. Indeed, if $C_{1}^{2}+C^{2}>0$, then $C_{1} u+C_{2} v$ is nontrivial solution of (3) and applying Lemma 1 we get a contradiction.

Therefore, (30) implies

$$
x^{(n-2)}(t)=-\int_{t}^{\infty} w(s, t) F(s) d s .
$$

Integrating $n-2$ times and using (32), we get

$$
x(t)=(-1)^{n-1} \int_{t}^{\infty} \int_{\tau_{n-2}}^{\infty} \cdots \int_{\tau_{1}}^{\infty} w\left(s, \tau_{1}\right) F(s) d s d \tau_{1} \cdots d \tau_{n-2}
$$

for $t \geq T_{x}$. Since $f \in C^{1}$, in view of the mean value theorem, there exists a function $\xi=\xi(s)$ such that

$$
f(x(s))=f^{\prime}(\xi(s)) x(s)
$$

and $0 \leq|\xi(s)| \leq|x(s)|$. We have for $x(s) \neq 0$

$$
f^{\prime}(\xi(s))=\frac{f(x(s))}{x(s)}
$$

thus, because $f \in C^{1}$, the function $f^{\prime}(\xi(s))$ is continuous and there exists $N>0$ such that

$$
\left|f^{\prime}(\xi(s))\right| \leq N
$$

Thus, from (33), we obtain

$$
\begin{aligned}
|x(t)| & \leq\left|\int_{t}^{\infty} \int_{\tau_{n-2}}^{\infty} \cdots \int_{\tau_{1}}^{\infty} w\left(s, \tau_{1}\right) r(s) f(x(s)) d s d \tau_{1} \cdots d \tau_{n-2}\right| \\
& =\left|\int_{t}^{\infty} \int_{\tau_{n-2}}^{\infty} \cdots \int_{\tau_{1}}^{\infty} w\left(s, \tau_{1}\right) r(s) f^{\prime}(\xi(s)) x(s) d s d \tau_{1} \cdots d \tau_{n-2}\right| .
\end{aligned}
$$

Hence, according to Lemma 2 with $F(t)=r(t) f^{\prime}(\xi(t)) x(t)$ we get for large $t$

$$
|x(t)| \leq M N \int_{t}^{\infty} s^{n-3}|r(s)||x(s)| d s
$$

By Lemma 4, we find that $x$ is identically zero for large $t$. Since $f \in C^{1}$, (34) and (35) imply (12), by Remark 1 every nontrivial solution $x$ of (1) is proper and so $x$ is identically zero for $t \geq T_{x}$. The proof is complete. 
Remark 3 If $f(u)=|u|^{\lambda} \operatorname{sgn} u, \lambda \geq 1$, then the assumption $f \in C^{1}(\mathbb{R})$ in Theorem 1 is satisfied. If $\lambda<1$, then $f \notin C^{1}(\mathbb{R})$ and Theorem 1 is not valid. The following example illustrates that assumptions of Theorem 1 are optimal.

Example 1 Consider the third order equation

$$
x^{\prime \prime \prime}(t)+x^{\prime}(t)+r(t)|x(t)|^{\lambda} \operatorname{sgn} x(t)=0, \quad t \geq 0,
$$

where $r(t)=\alpha(\alpha+1)(\alpha+2)(t+1)^{\alpha \lambda-\alpha-3}+\alpha(t+1)^{\alpha \lambda-\alpha-1}$ and $\alpha>0$.

If $\lambda<1$, then $\int_{0}^{\infty} r(t) d t<\infty$, i.e. condition (13) is satisfied, however, we have $f \notin C^{1}(\mathbb{R})$. If $\lambda \geq 1$, condition (13) is not satisfied.

One can check that in both cases $x(t)=(t+1)^{-\alpha}$ is a solution vanishing at infinity of (36). This shows the strictness of both assumptions of Theorem 1 , that is, $f \in C^{1}(\mathbb{R})$ and (13).

\section{Oscillation in the linear case}

In this section we prove the existence of oscillatory solutions of (2), which are bounded and not vanishing at infinity.

Theorem 2 Let $n \geq 3$, u be a nontrivial solution of (3) and

$$
\int_{0}^{\infty} s^{n-3}\left|q^{\prime}(s)\right| d s<\infty
$$

Then (2) has an oscillatory solution $\phi$ such that

$$
\phi(t)= \begin{cases}u^{\prime}(t)+\varepsilon(t) & \text { for } n \text { odd } \\ u(t)+\varepsilon(t) & \text { for } n \text { even }\end{cases}
$$

where $\varepsilon$ is a continuous function on $[0, \infty)$ and $\lim _{t \rightarrow \infty} \varepsilon(t)=0$. In particular,

$$
0<\limsup _{t \rightarrow \infty}|\phi(t)|<\infty
$$

To prove this theorem, we give asymptotic expressions of the integrals in (8).

Lemma 5 Let $n \geq 3$ and (37) hold. If $u$ is a nontrivial (oscillatory) solution of (3), then there exist constants $c_{i}, i=0,1, \ldots, n-2, c_{n-2} \neq 0$, and a function $\varepsilon$ such that

$$
\Gamma_{u}(t)= \begin{cases}\sum_{i=0}^{n-3} c_{i} t^{i}+c_{n-2} u^{\prime}(t)+\varepsilon(t) & \text { for nodd }, \\ \sum_{i=0}^{n-3} c_{i} t^{i}+c_{n-2} u(t)+\varepsilon(t) & \text { for n even },\end{cases}
$$

where $\varepsilon$ is a continuous function on $[0, \infty)$ and $\lim _{t \rightarrow \infty} \varepsilon(t)=0$.

Proof In view of $(\mathrm{H})$, we have $\lim _{t \rightarrow \infty} q(t)=q_{\infty}, 0<q_{\infty}<\infty$. Let $\mu$ be an integer, $\mu \geq 1$. Using (3) we get

$$
\begin{aligned}
\int_{0}^{t} \frac{u(s)}{q^{\mu-1}(s)} d s & =-\int_{0}^{t} \frac{u^{\prime \prime}(s)}{q^{\mu}(s)} d s=-\frac{u^{\prime}(t)}{q^{\mu}(t)}+\frac{u^{\prime}(0)}{q^{\mu}(0)}-\mu \int_{0}^{t} \frac{q^{\prime}(s) u^{\prime}(s)}{q^{\mu}(s)} d s \\
& =\alpha-\left(q_{\infty}\right)^{-\mu} u^{\prime}(t)+\varepsilon_{0}(t)
\end{aligned}
$$


where

$$
\begin{aligned}
& \alpha=\frac{u^{\prime}(0)}{q^{\mu}(0)}-\mu \int_{0}^{\infty} \frac{q^{\prime}(s) u^{\prime}(s)}{q^{\mu}(s)} d s, \\
& \varepsilon_{0}(t)=\mu \int_{t}^{\infty} \frac{q^{\prime}(s)}{q^{\mu}(s)} d s+\left(\left(q_{\infty}\right)^{-\mu}-(q(t))^{-\mu}\right) u^{\prime}(t)
\end{aligned}
$$

and $\lim _{t \rightarrow \infty} \varepsilon_{0}(t)=0$.

Similarly, let $v$ be an integer, $v \geq 1$. Using (3) we have

$$
\begin{aligned}
\int_{0}^{t} \frac{(t-s) u(s)}{q^{v-1}(s)} d s= & -\int_{0}^{t} \frac{(t-s) u^{\prime \prime}(s)}{q^{v}(s)} d s \\
= & \frac{u^{\prime}(0)}{q^{v}(0)} t-\frac{u(t)}{q^{v}(t)}+\frac{u(0)}{q^{v}(0)}-v \int_{0}^{t} \frac{q^{\prime}(s) u(s)}{q^{v+1}(s)} d s \\
& -v \int_{0}^{t} \frac{(t-s) q^{\prime}(s) u^{\prime}(s)}{q^{v+1}(s)} d s \\
= & \beta t+\gamma-\left(q_{\infty}\right)^{-v} u(t)+\varepsilon_{1}(t),
\end{aligned}
$$

where

$$
\begin{aligned}
& \beta=\frac{u^{\prime}(0)}{q^{v}(0)}-v \int_{0}^{\infty} \frac{q^{\prime}(s) u^{\prime}(s)}{q^{v+1}(s)} d s, \\
& \gamma=\frac{u(0)}{q^{\nu}(0)}+v \int_{0}^{\infty} \frac{q^{\prime}(s) s u^{\prime}(s)}{q^{v+1}(s)} d s-v \int_{0}^{\infty} \frac{q^{\prime}(s) u(s)}{q^{v+1}(s)} d s, \\
& \varepsilon_{1}(t)=v \int_{t}^{\infty} \frac{q^{\prime}(s) u(s)}{q^{v+1}(s)} d s+v \int_{t}^{\infty} \frac{q^{\prime}(s)(t-s) u^{\prime}(s)}{q^{v+1}(s)} d s+\left(\left(q_{\infty}\right)^{-v}-(q(t))^{-v}\right) u(t)
\end{aligned}
$$

and $\lim _{t \rightarrow \infty} \varepsilon_{1}(t)=0$.

When $n=3$, the assertion follows from (38) with $\mu=1$. Similarly, when $n=4$, the assertion follows from (39) with $v=1$.

Now, let $n \geq 5$. Let $j$ be an integer and $n-3 \geq j \geq 2$. Fixed $k>0$ and integrating by parts, we obtain

$$
\begin{aligned}
\int_{0}^{t} \frac{(t-s)^{j}}{q^{k}(s)} u(s) d s & =-\int_{0}^{t} \frac{(t-s)^{j} u^{\prime \prime}(s)}{q^{k+1}(s)} d s \\
& =\frac{u^{\prime}(0)}{q^{k+1}(0)} t^{j}-j \int_{0}^{t} \frac{(t-s)^{j-1} u^{\prime}(s)}{q^{k+1}(s)} d s-(k+1) \int_{0}^{t} \frac{(t-s)^{j} q^{\prime}(s) u^{\prime}(s)}{q^{k+2}(s)} d s .
\end{aligned}
$$

Similarly

$$
\int_{0}^{t} \frac{(t-s)^{j-1} u^{\prime}(s)}{q^{k+1}(s)} d s=\frac{-u(0)}{q^{k+1}(0)} t^{j-1}+(j-1) \int_{0}^{t} \frac{(t-s)^{j-2} u(s)}{q^{k+1}(s)} d s .
$$

Applying Lemma 1 and (37), we have

$$
\begin{aligned}
& \int_{0}^{\infty} \frac{(t-s)^{j-1} q^{\prime}(s) u(s)}{q^{k+2}(s)} d s=\sum_{m=0}^{j-1} d_{m} t^{m}, \\
& \int_{0}^{\infty} \frac{(t-s)^{j} q^{\prime}(s) u^{\prime}(s)}{q^{k+2}(s)} d s=\sum_{m=0}^{j} \bar{d}_{m} t^{m},
\end{aligned}
$$


where

$$
\begin{aligned}
& d_{m}=(-1)^{j-m-1}\left(\begin{array}{c}
j-1 \\
m
\end{array}\right) \int_{0}^{\infty} \frac{q^{\prime}(s)}{q^{k+2}(s)} s^{j-m-1} u(s) d s, \\
& \bar{d}_{m}=(-1)^{j-m}\left(\begin{array}{c}
j \\
m
\end{array}\right) \int_{0}^{\infty} \frac{q^{\prime}(s)}{q^{k+2}(s)} s^{j-m} u^{\prime}(s) d s .
\end{aligned}
$$

Hence

$$
\int_{0}^{t} \frac{(t-s)^{j} u(s)}{q^{k}(s)} d s=\sum_{m=0}^{j} D_{m} t^{m}-j(j-1) \int_{0}^{t} \frac{(t-s)^{j-2} u(s)}{q^{k+1}(s)} d s+H_{j}(t)
$$

where $D_{j}$ are constants and

$$
H_{j}(t)=(k+1) \int_{t}^{\infty} \frac{(t-s)^{j-1} q^{\prime}(s)}{q^{k+2}(s)}\left(j u(s)+(t-s) u^{\prime}(s)\right) d s .
$$

Since

$$
\left|H_{j}(t)\right| \leq(k+1) \int_{t}^{\infty} \frac{s^{j-1}\left|q^{\prime}(s)\right|}{q^{k+2}(s)}\left(j|u(s)|+s\left|u^{\prime}(s)\right|\right) d s,
$$

in virtue of Lemma 1 and (37), we get $\lim _{t \rightarrow \infty} H_{j}(t)=0$.

Let $n$ be odd, $n \geq 5$. By using recursively (40) with

$$
j=\{2,4,6, \ldots, n-3\} \text { and } k=(n-3-j) / 2 \text {, }
$$

we obtain the following estimation for the function $\Gamma_{u}$ given by (9):

$$
\Gamma_{u}(t)=\sum_{i=0}^{n-3} c_{i} t^{i}-(n-3) ! \int_{0}^{t} \frac{u(s)}{q^{(n-3) / 2}(s)} d s+K_{1}(t),
$$

where $c_{i}$ are suitable constants and $K_{1}$ is a continuous function on $[0, \infty)$ and $\lim _{t \rightarrow \infty} K_{1}(t)=0$.

Choosing $\mu=(n+1) / 2$ in (38), from (41) the assertion follows for $n$ odd, $n \geq 5$.

Finally, let $n$ be even, $n \geq 6$. By using a similar argument to the one above given and applying recursively (40) with

$$
j=\{3,5,7, \ldots, n-3\} \quad \text { and } \quad k=(n-3-j) / 2 \text {, }
$$

we obtain the following estimation for the function $\Gamma_{u}$ :

$$
\Gamma_{u}(t)=\sum_{i=0}^{n-3} \bar{c}_{i} t^{i}-(n-3) ! \int_{0}^{t} \frac{(t-s) u(s)}{q^{n / 2-2}(s)} d s+K_{2}(t)
$$

where $\bar{c}_{i}$ are suitable constants and $K_{2}$ is a continuous function on $[0, \infty)$ and $\lim _{t \rightarrow \infty} K_{2}(t)=0$.

Choosing $v=(n-2) / 2$ in (39), from (42) the assertion follows. 
Proof of Theorem 2 By Lemma 5, (2) has a solution $u^{\prime}(t)+\varepsilon(t)$ if $n$ is odd, and $u(t)+\varepsilon(t)$ if $n$ is even, where $\lim _{t \rightarrow \infty} \varepsilon(t)=0$. According to Lemma 1 this solution is oscillatory, bounded, and not vanishing at infinity.

\section{Oscillation in the nonlinear case}

Our main results are given by the following.

Theorem 3 Let $n \geq 3$ and $u$ be a nontrivial solution of (3).

Assume (37) and

$$
\int_{0}^{\infty} t^{n-3}|r(t)| d t<\infty
$$

Then for any real numbers $c_{0}, c_{1}$, (1) has a solution $x$, defined on $\left[T_{x}, \infty\right), T_{x} \geq 0$, such that

$$
x(t)= \begin{cases}c_{0}+c_{1} u^{\prime}(t)+\varepsilon(t) & \text { for } n \text { odd } \\ c_{0}+c_{1} u(t)+\varepsilon(t) & \text { for n even }\end{cases}
$$

where $\varepsilon$ is a continuous function on $\left[T_{x}, \infty\right)$ and $\lim _{t \rightarrow \infty} \varepsilon(t)=0$.

Consequently, (1) has oscillatory solutions $x$ such that

$$
0<\limsup _{t \rightarrow \infty}|x(t)|<\infty
$$

Proof By Theorem 2, (2) has an oscillatory solution $\phi$, which is bounded and not vanishing at infinity. Applying Theorem A with $j=0$, (1) has a solution with the same asymptotic properties as that one of (2).

Theorem 4 Let $n \geq 3$ and $u$, $v$ be two linearly independent solutions of (3). Assume (37) and for any positive constant $\mu$

$$
\int_{0}^{\infty} t^{n-3} \bar{f}\left(\mu t^{n-3}\right)|r(t)| d t<\infty
$$

where the function $\bar{f}$ is defined in the Preliminaries.

Then for any vector $\left(c_{0}, c_{1}, \ldots, c_{n-1}\right) \in \mathbb{R}^{n}$ there exists a solution $x$ of $(1)$, defined on $\left[T_{x}, \infty\right)$, $T_{x} \geq 0$, such that

$$
x(t)= \begin{cases}\sum_{i=0}^{n-3} c_{i} t^{i}+c_{n-2} u^{\prime}(t)+c_{n-1} v^{\prime}(t)+\varepsilon(t) & \text { for } n \text { odd }, \\ \sum_{i=0}^{n-3} c_{i} t^{i}+c_{n-2} u(t)+c_{n-1} v(t)+\varepsilon(t) & \text { for } n \text { even },\end{cases}
$$

where $\varepsilon$ is a continuous function on $\left[T_{x}, \infty\right)$ and $\lim _{t \rightarrow \infty} \varepsilon(t)=0$.

If, in addition, $f \in C^{1}(\mathbb{R})$ and there exists $M>0$ such that

$$
\left|f^{\prime}(u)\right| \leq M \bar{f}(u) \quad \text { for large }|u|
$$

then the solution $x$ given by (45) is unique. 
Proof Existence. As noticed above, the set (8) is a basis for the space of solutions of (2). Applying Theorem A with $j=n-3$ and Theorem 3, (1) has a solution $x$ satisfying (45).

Uniqueness. For the sake of simplicity, let $n$ be even. The case $n$ odd follows in a similar way. Suppose, by contradiction, that for $\left(c_{0}, c_{1}, \ldots, c_{n-1}\right) \in \mathbb{R}^{n}$ there exist two different solutions $x$ and $\bar{x}$ of (1) satisfying (45). Then for $\bar{z}(t)=x(t)-\bar{x}(t)$ we have

$$
\lim _{t \rightarrow \infty} \bar{z}(t)=0
$$

and

$$
\bar{z}^{(n)}(t)+q(t) \bar{z}^{(n-2)}(t)+r(t)(f(x(t))-f(\bar{x}(t)))=0 .
$$

In view of the mean value theorem, there exists a function $\xi=\xi(s)$ such that

$$
f(x(t))-f(\bar{x}(t))=f^{\prime}(\xi(t))(x(t)-\bar{x}(t))
$$

and

$$
\min \{x(t), \bar{x}(t)\} \leq \xi(t) \leq \max \{x(t), \bar{x}(t)\} .
$$

Then we have

$$
|\xi(t)| \leq M_{1} t^{n-3}
$$

Therefore $\bar{z}$ is a solution of

$$
z^{(n)}(t)+q(t) z^{(n-2)}(t)+R(t) z(t)=0,
$$

where $R(t)=r(t) f^{\prime}(\xi(t))$, for $t \geq 1$ and a suitable constant $M_{1}>0$.

Let (46) hold for $|u| \geq u_{0}>0$. Then, in view of (44), the condition (13) for (48) is satisfied, because

$$
\int_{0}^{\infty} t^{n-3}|R(t)| d t \leq \int_{0}^{\infty} t^{n-3}|r(t)|\left(M_{2}+M \bar{f}\left(M_{1} t^{n-3}\right)\right) d t<\infty,
$$

where $M_{2}=\max _{|v| \leq u_{0}}\left|f^{\prime}(v)\right|$. Hence we can apply Theorem 1 to (48), which gives a contradiction with (47).

Remark 4 Theorem 3 extends [1, Theorem 1.4] and Theorem 4 [1, Theorem 1.3] stated for (1) with $r(t)>0$.

An application of Theorems 3 and 4 is the following. Consider the Emden-Fowler type equation

$$
x^{(n)}(t)+x^{(n-2)}(t)+r(t)|x(t)|^{\lambda} \operatorname{sgn} x(t)=0,
$$

where $\lambda>0$. Then (46) is satisfied for any $\lambda>0$ and (44) reads

$$
\int_{0}^{\infty} t^{(n-3)(\lambda+1)}|r(t)| d t<\infty
$$


Thus, according to Theorem 4 , for a fixed vector $\left(c_{0}, c_{1}, \ldots, c_{n-1}\right)$ there exists a unique solution of (49) which has the asymptotic representation (45).

Now, consider (49), where $n$ is even, $r(t)>0$ for $t \geq 0$ and $\lambda \neq 1$. By [1, Corollary 1.6], if $\int^{\infty} t^{n-3} r(t) d t=\infty$, then every solution of (49) is oscillatory. Obviously, condition (37) is satisfied. Therefore, the condition (43) in Theorem 3 is optimal.

From this and Theorem 3 we get the following.

Corollary 1 Let $\lambda>1, n$ even, $n \geq 4$ and $r(t)>0$ for $t \geq 0$. Then (49) has oscillatory solutions.

\section{Asymptotic equivalence of linear equations}

In this section we present another consequence of our results.

Consider the linear equation

$$
x^{(n)}(t)+q(t) x^{(n-2)}(t)+r(t) x(t)=0, \quad n \geq 3,
$$

and denote by $S_{x}$ and $S_{y}$ the solution space of (5) and (2), respectively.

We say that (2) and (5) are asymptotically equivalent, if there exists a map $T: S_{y} \rightarrow S_{x}$ such that for every $y \in S_{y}$ there exists a unique $x \in S_{x}$ such that $T(y)=x$ and

$$
\lim _{t \rightarrow \infty}(x(t)-y(t))=0 .
$$

Applying Theorem A and Theorem 1 we get the following.

Theorem 5 Assume $n \geq 3$ and

$$
\int_{0}^{\infty} t^{2 n-6}|r(t)| d t<\infty
$$

Then (2) and (5) are asymptotically equivalent.

Proof As we noticed above, functions $t^{j}, \Gamma_{u}$ and $\Gamma_{v}$ are linearly independent solutions of (2).

By Theorem A, there exist functions $\eta_{j}, j=0, \ldots, n-1$, which tend to zero as $t \rightarrow \infty$, such that

$$
\begin{aligned}
& x_{j}(t)=t^{j}+\eta_{j}(t), \quad j=0, \ldots, n-3, \\
& x_{n-2}(t)=\Gamma_{u}(t)+\eta_{n-2}(t), \\
& x_{n-1}(t)=\Gamma_{v}(t)+\eta_{n-1}(t)
\end{aligned}
$$

are solutions of (5). Hence, applying again Theorem A, we get

$$
\lim _{t \rightarrow \infty} \eta_{j}^{(i)}(t)=0, \quad i=1, \ldots, n-1 .
$$

We show that $\eta_{j}$ are uniquely determined. Without loss of generality, assume by contradiction that there exist $\eta_{0}$ and $\bar{\eta}_{0}$ such that $\eta_{0} \not \equiv \bar{\eta}_{0}$ and

$$
x_{0}(t)=1+\eta_{0}(t), \quad \bar{x}_{0}(t)=1+\bar{\eta}_{0}(t)
$$


are solutions of (5). Then $\bar{x}_{0}-x_{0}$ is also a solution of (5) and tends to zero as $t \rightarrow \infty$. This is a contradiction with Theorem 1 . For $j \in\{1, \ldots, n-1\}$ we proceed by the same way.

A standard argument shows that solutions $x_{j}, j=0, \ldots, n-1$, are linearly independent. Set

$$
z(t)=\sum_{j=0}^{n-1} c_{j} x_{j}(t)
$$

and let us prove that $z(t) \equiv 0$ implies that $\left(c_{0}, c_{1}, \ldots, c_{n-1}\right)$ is the zero vector in $\mathbb{R}^{n}$. From (53) we obtain

$$
0 \equiv \frac{d^{n-2}}{d t^{n-2}} z(t)=c_{n-2} u(t)+c_{n-1} v(t)+c_{n-2} \eta_{n-2}^{(n-2)}(t)+c_{n-1} \eta_{n-1}^{(n-2)}(t)
$$

From this, (52) with $i=n-2$ and Lemma 1, we get $c_{n-2}=c_{n-1}=0$. Therefore,

$$
z(t)=\sum_{j=0}^{n-3} c_{j} x_{j}(t) .
$$

Since

$$
0 \equiv \frac{d^{n-3}}{d t^{n-3}} z(t)=c_{n-3}+\eta_{n-3}^{(n-3)}(t)
$$

and $\lim _{t \rightarrow \infty} \eta_{n-3}^{(n-3)}(t)=0$, we have $c_{n-3}=0$. Proceeding in the same way we get $c_{i}=0$ for $i=0, \ldots, n-2$.

Denote

$$
\begin{aligned}
& \bar{S}_{x}=\left\{x_{j}(t), j=0, \ldots, n-1\right\}, \\
& \bar{S}_{y}=\left\{t^{j}, j=0, \ldots, n-3, \Gamma_{u}(t), \Gamma_{\nu}(t)\right\} .
\end{aligned}
$$

Since $\eta_{j}$ are uniquely determined, there exists a 1-1 map between the sets $\bar{S}_{x}$ and $\bar{S}_{y}$ and so the assertion follows.

Remark 5 The assumption (37) is not needed in Theorem 5.

\section{Open problems}

(1) Does Corollary 1 hold for $n$ even and $\lambda \leq 1$ ?

(2) Let $q$ be bounded, but not of bounded variation on $[0, \infty)$. Then (3) can have unbounded oscillatory solutions, see, e.g., [17, Chapter VI-5, Theorem 3]. Similarly, if (3) is oscillatory and $\lim _{t \rightarrow \infty} q(t)=0$, then (3) can have again unbounded oscillatory solutions, as the Euler equation illustrates. In these cases, it should be interesting to find conditions under which (1) has unbounded oscillatory solutions too.

(3) Let $q$ be unbounded. Thus, as the Armellini-Tonelli-Sansone theorem shows, (3) can have (oscillatory) solutions which tend to zero as $t \rightarrow \infty$, see [20] for a detailed survey on this topic. In particular, in $[20,21]$ the boundedness and the existence of vanishing at infinity solutions are investigated for second order linear equations with advanced 
arguments. As before, also in this situation, it should be an interesting problem studying asymptotic properties of possible oscillatory solutions of (1). Note that for (1) with $n=4$, conditions which ensure that all solutions are oscillatory, can be found in $[6,7]$ or in $[8$, Theorem 4].

(4) Let $g$ be a continuous function on $\mathbb{R}$ such that

$$
\lim _{|u| \rightarrow \infty} \int_{0}^{u} g(\sigma) d \sigma=\infty
$$

Under assumptions $(\mathrm{H})$, any solution of the nonlinear equation

$$
y^{\prime \prime}(t)+q(t) g(y(t))=0
$$

is bounded together with its first derivative, see, e.g., [18, Theorem 4]. Thus, motivated by the results here obtained, we can ask under which conditions the nonlinear equation

$$
x^{(n)}(t)+q(t) g\left(x^{(n-2)}(t)\right)+r(t) f(x(t))=0
$$

has oscillatory bounded solutions.

(5) Recently, oscillation of equations with a forcing term have been studied. For example, the boundedness of any solutions is studied in [15] and the periodic case in [14]. Moreover, in [16] a two-term equation with forcing term $e$ has been considered and the oscillation is studied under additional conditions on the function $r$. Thus, it seems interesting to extend our study to the existence of oscillatory solutions and solutions vanishing at infinity for the equation with the forcing term

$$
x^{(n)}(t)+q(t) x^{(n-2)}(t)+r(t) f(x(t))=e(t) .
$$

\section{Competing interests}

The authors declare that they have no competing interests.

Authors' contributions

All authors contributed equally to the manuscript and read and approved the final draft.

\section{Author details}

'Department of Mathematics and Statistics, Faculty of Science, Masaryk University, Kotlářská 2, Brno, 611 37, Czech Republic. 'Department of Mathematics and Informatics 'Ulisse Dini', University of Florence, Florence, 50139, Italy.

\section{Acknowledgements}

The first and second authors were supported by the grant GAP201/11/0768 of the Czech Grant Agency. The authors thank both referees for their valuable comments to the paper.

Received: 25 November 2013 Accepted: 12 February 2014 Published: 26 Feb 2014

\section{References}

1. Kiguradze, IT: An oscillation criterion for a class of ordinary differential equations. Differ. Uravn. 28, 201-214 (1992)

2. Kiguradze, IT, Chanturia, TA: Asymptotic Properties of Solutions of Nonautonomous Ordinary Differential Equations. Kluwer Academic, Dordrecht (1993)

3. Bartušek, M, Cecchi, M, Došlá, Z, Marini, M: Oscillation for third-order nonlinear differential equations with deviating argument. Abstr. Appl. Anal. 2010, Article ID 278962 (2010). doi:10.1155/2010/278962

4. Bartušek, M, Cecchi, M, Došlá, Z, Marini, M: Positive solutions of third order damped nonlinear differential equations. Math. Bohem. 136, 205-213 (2011)

5. Graef, JR, Saker, SH: Oscillation theory of third-order nonlinear functional differential equations. Hiroshima Math. J. 43, 49-72 (2013)

6. Amara, JB: Oscillation criteria for fourth-order differential equations with middle term. Math. Nachr. 285, 42-46 (2012) 
7. Amara, JB: Comparison theorem for conjugate points of a fourth-order linear differential equation. Can. Math. Bull. 56, 39-43 (2013)

8. Bartušek, M, Došlá, Z: Asymptotic problems for fourth order nonlinear differential equations. Bound. Value Probl. 2013, 89 (2013). doi:10.1186/1687-2770-2013-89

9. Bartušek, M, Cecchi, M, Došlá, Z, Marini, M: Unbounded solutions of third order delayed differential equations with damping term. Cent. Eur. J. Math. 9, 184-195 (2011)

10. Bartušek, M, Cecchi, M, Došlá, Z, Marini, M: On fourth order differential equation with deviating argument. Abstr. Appl. Anal. 2012, Article ID 185242 (2012). doi:10.1155/2012/185242

11. Astashova, IV: On the asymptotic behavior at the infinity of solutions to quasi-linear differential equations. Math. Bohem. 135, 373-382 (2010)

12. Astashova, IV: On existence of non-oscillatory solutions to quasi-linear differential equations. Georgian Math. J. 14 223-238 (2007)

13. Bartušek, M, Cecchi, M, Došlá, Z, Marini, M: Asymptotics for higher order differential equations with a middle term. J. Math. Anal. Appl. 388, 1130-1140 (2012)

14. Kiguradze, IT, Lomtatidze, A: Periodic solutions of nonautonomous ordinary differential equations. Monatshefte Math. $159,235-252(2010)$

15. Perov, Al: On bounded solutions of $n$-order nonlinear ordinary differential equations. Differ. Uravn. 46, 1226-1244 (2010). Translation: Differ. Equ. 46, 1236-1252 (2010)

16. Sun, YG, Han, Z: On forced oscillation of higher-order functional differential equations. Appl. Math. Comput. 218, 6966-6971 (2012)

17. Bellman, R: Stability Theory of Differential Equations. McGraw-Hill, New York (1953)

18. Marini, M: Criteri di limitatezza per le soluzioni dell'equazione lineare del secondo ordine. Boll. Unione Mat. Ital. 11, 154-165 (1975)

19. Agarwal, RP, Grace, SR, O'Regan, D: Oscillation Theory for Second Order Linear, Half-Linear, Superlinear and Sublinear Dynamic Equations. Kluwer Academic, Dordrecht (2003)

20. Došlá, Z, Kiguradze, IT: On vanishing at infinity solutions of second order linear differential equations with advanced arguments. Funkc. Ekvacioj 41, 189-205 (1998)

21. Došlá, Z, Kiguradze, IT: On boundedness and stability of solutions of second order linear differential equations with advanced arguments. Adv. Math. Sci. Appl. 9, 1-24 (1999)

10.1186/1687-2770-2014-48

Cite this article as: Bartušek et al.: Oscillation for higher order differential equations with a middle term. Boundary Value Problems 2014, 2014:48

\section{Submit your manuscript to a SpringerOpen ${ }^{\circ}$ journal and benefit from:}

- Convenient online submission

- Rigorous peer review

Immediate publication on acceptance

Open access: articles freely available online

- High visibility within the field

- Retaining the copyright to your article 